\title{
Análise de rentabilidade de irrigação na cana-de-açúcar: estudo de caso de uma usina de Goiás
}

Irrigation profitability analysis in sugarcane: case study of a sugarcane mill from Goiás

\author{
Flávio Henrique Campos ${ }^{1 *}$; Aline Bigaton 2; Haroldo José Torres da Silva3; Pedro \\ Valentim Marques ${ }^{4}$; Rubens Duarte Coelho ${ }^{5}$ \\ 1 Escola Superior de Agricultura "Luiz de Queiroz", Universidade de São Paulo - Bacharel em Engenharia \\ Agronômica - Rua Alexandre Herculano 120, sala B12, Vila Monteiro - CEP 13418-445 - Piracicaba (SP), \\ Brasil \\ 2 Universidade de Campinas - Mestranda em Planejamento de Sistemas Energéticos (Faculdade de \\ Engenharia Mecânica) - Rua Alexandre Herculano 120, sala B12, Vila Monteiro - CEP 13418-445 - \\ Piracicaba (SP), Brasil \\ ${ }^{3}$ Escola Superior de Agricultura "Luiz de Queiroz", Universidade de São Paulo - Doutorando em \\ Economia Aplicada (Departamento de Economia, Administração e Sociologia) - Rua Alexandre Herculano \\ 120, sala B12, Vila Monteiro - CEP 13418-445 - Piracicaba (SP), Brasil \\ ${ }^{4}$ Escola Superior de Agricultura "Luiz de Queiroz", Universidade de São Paulo - Professor Titular - \\ Departamento de Economia, Administração e Sociologia - Rua Alexandre Herculano 120, sala T4, Vila \\ Monteiro - CEP 13418-445 - Piracicaba (SP), Brasil \\ ${ }^{5}$ Escola Superior de Agricultura "Luiz de Queiroz", Universidade de São Paulo - Professor Titular - \\ Departamento de Engenharia de Biossistemas - Av. Pádua Dias, 11 - CEP 13418-900 - Piracicaba (SP), \\ Brasil
}

\section{Resumo}

Esta publicação apresenta uma análise financeira do sistema de irrigação por sistema linear móvel praticado por uma usina localizada no estado de Goiás, com o intuito de fornecer dados para alimentar um modelo que poderá ser utilizado posteriormente como uma ferramenta de análise econômica e de rentabilidade da irrigação na cana-de-açúcar. Foram avaliados os custos fixos e variáveis anuais e os benefícios diretos e indiretos da irrigação. O benefício direto advém do aumento da receita pelo aumento de produtividade da área irrigada e aumento da longevidade do canavial; e os benefícios indiretos são provenientes da redução de custos decorrentes da redução da área de produção e dos custos de colheita e transporte. Como resultado, a irrigação apresentou custos totais anuais de aproximadamente $R \$ 72,00$ por tonelada. A relação benefício/custo da irrigação foi de 1,066, considerando somente o benefício direto de produtividade (Etapa 1) e de 1,313 ao considerar o benefício direto do aumento da longevidade e da produtividade (Etapa 2). Portanto, a irrigação apresenta uma boa rentabilidade financeira nesse caso estudado.

Palavras-chave: matéria-prima, produtividade, sucroenergético, tecnologias

\section{Abstract}

This paper presents a financial analysis of a linear mobile pivot irrigation system practiced by a plant located in the state of Goiás, Brasil, in order to provide data to feed a model that can be used later as a tool of economic and profitability analysis of sugarcane irrigation. The fixed costs, annual variables costs and the direct and indirect benefits of irrigation were evaluated. The direct benefit came from the increased revenue by productivity gains on irrigated areas and increased longevity of the cane field. The indirect benefits came from costs reduction due to the reduction of the area of production and of the costs of harvesting and transportation. As a result, the irrigation presented total annual costs of approximately $R \$ 72.00$ per ton. The benefit cost ratio of irrigation with increased productivity was 1,066 in the first stage and 1,313 in the second stage, which includes productivity and longevity increase. Therefore, irrigation presented a good financial profitability in this study.

Keywords: raw material, productivity, sugar-energy industry, technologies 


\section{Introdução}

A produção de cana-de-açúcar no Brasil possui grande importância para o país em termos econômicos, sociais e ambientais. De acordo com o último boletim de acompanhamento de safra (CONAB, 2017), estima-se que na safra 2017/18 haverá uma produção de 647,6 milhões de toneladas e 8,84 milhões de área colhida. Para a região Centro-oeste, estima-se manutenção da área colhida e da produtividade, em relação à safra passada, com produção de 136,17 milhões de toneladas, aumento de 1,4\%.

De acordo com o levantamento do grupo Programa de Educação Continuada em Economia e Gestão de Empresas [PECEGE] (2015), os custos totais de produção de cana-de-açúcar foram superiores ao preço potencial que seria pago pela cana própria, caso esta fosse remunerada pela respectiva quantidade e valor de Açúcar Total Recuperável [ATR] $\left(R \$ \mathrm{Kg}^{-1}\right)$, isto é, para as usinas, a aquisição de cana de fornecedores apresenta-se mais atraente financeiramente do que a produção própria. Além disso, os custos de produção agroindustriais têm apresentado crescimento real ao longo dos anos e o preço de seus produtos (açúcar e etanol) foram pouco atraentes ao longo das safras passadas. A safra atual traz uma perspectiva de melhoria nos preços de venda desses produtos, motivados especialmente pelo aumento do preço do açúcar. Porém, o histórico recente de baixa rentabilidade do setor tornou evidente a necessidade de se buscar alternativas que possam inovar o processo produtivo.

Segundo Marques e Frizzone (2005), a irrigação apresentou-se como uma tecnologia para aumentar a produtividade e a estabilidade da produção agrícola, possibilitando a redução do risco produtivo. No entanto, houve um elevado risco econômico na adoção dessa tecnologia devido ao elevado investimento inicial, e devido a diversos fatores importantes no seu resultado econômico, como, variação do preço do produto, vida útil, taxa de juros, produtividade, custo dos insumos (especialmente a energia) etc.

O estado de Goiás apresenta crescente importância no desenvolvimento da agricultura nacional. Segundo estimativa (CONAB, 2016), o estado de Goiás é o segundo maior produtor de cana-de-açúcar na safra 2016/17 com 10,4\% da cana total produzida, abaixo somente do estado de São Paulo com $56,2 \%$ da produção total (Figura 1). 


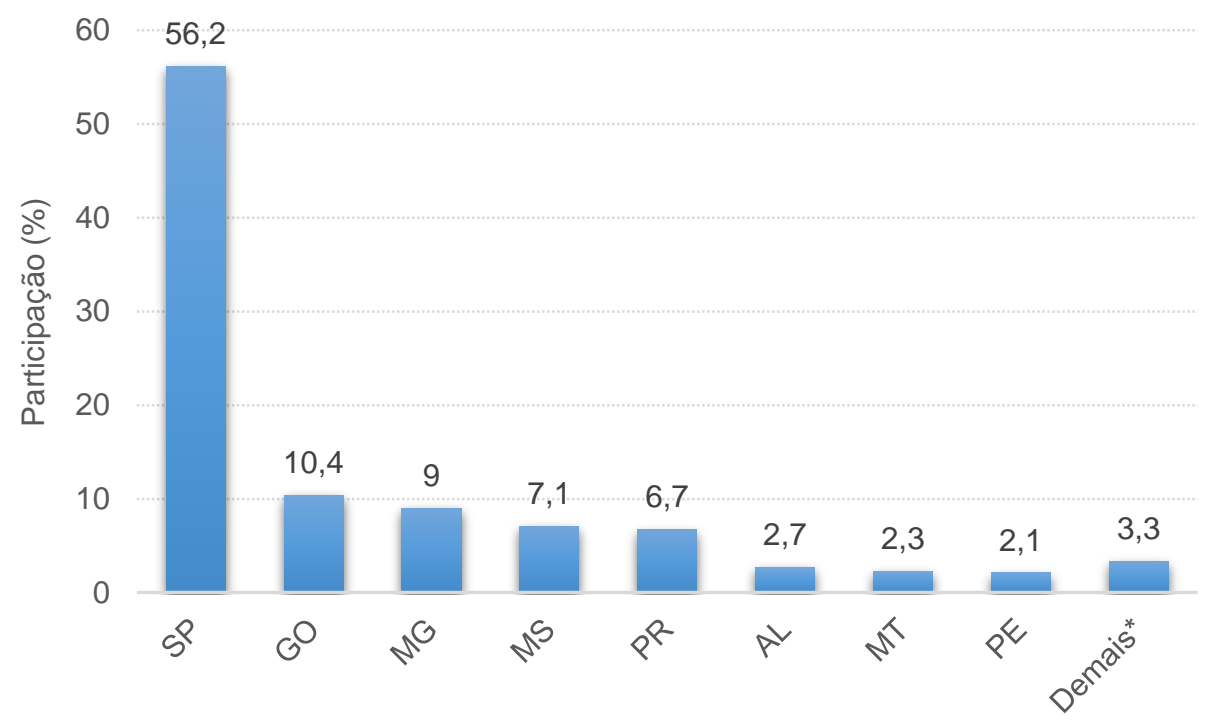

Figura 1. Participação estimada na produção de cana-de-açúcar por estado Nota: estimativa realizada em agosto/2016

Fonte: CONAB (2016)

A redução das chuvas no ano safra 2016/17 impactou negativamente a produtividade, devido a uma seca prolongada e antecipada. Em contraposição, essa estiagem favoreceu o índice de ATR para valores estimados de $144,4 \mathrm{~kg} \mathrm{t}^{-1}$ na atual safra, acréscimo de 8,1\% em relação à safra passada (CONAB, 2016). No entanto, foram registradas geadas no estado que irão impactar negativamente a produção e a qualidade da cana em áreas sem nenhum histórico anterior de danos por geada.

A produção agropecuária está sujeita a diversos riscos ao longo da cadeia de produção. $\mathrm{Na}$ fase agrícola, os riscos são elevados devido aos fatores externos de produção de difícil controle pelo homem, como fatores climáticos (Agrológica, 2002). A irrigação permite uma redução desses riscos produtivos. Há, no entanto, outro tipo de risco que se evidencia nos sistemas irrigados, o risco econômico.

Segundo Frizzone et al. (2005), o risco do sistema de irrigação está associado a sua elevada vida útil do sistema, o que dificulta a estimativa do valor econômico do projeto devido a suas variações ao longo do tempo. Adicionalmente, os investimentos iniciais na irrigação [CAPEX] tendem a ser elevados. Esse fator apresenta-se como uma barreira para a decisão de irrigar, dificultando para os produtores de menor caixa e exigindo que a avaliação do projeto econômico analise rigorosamente o fluxo de caixa e o cronograma do projeto. Diversos fatores associados ao projeto estão sujeitos a variação ao longo da sua vida útil e possuem impacto significativo na análise. Conforme análise de sensibilidade conduzida em modelo computacional (Marques e Frizzone, 2005), os fatores de maior influência para o benefício líquido anual da irrigação de canade-açúcar em Piracicaba, São Paulo, foram, em ordem decrescente: valor de venda do 
produto, vida útil, taxa de manutenção, taxa de juros, preço da água e mão de obra necessária.

Dessa forma, desenvolveu-se o estudo de caso para uma usina produtora de açúcar, etanol e bioeletricidade do estado de Goiás, afim de analisar a rentabilidade de um sistema de irrigação implantado para a produção de cana-de-açúcar em uma usina, avaliando os principais custos fixos do sistema e sua depreciação anual, assim como os custos variáveis nos sistemas de irrigação e os benefícios diretos e indiretos ocorridos da irrigação em comparação à produção em sequeiro.

O desenvolvimento desse estudo foi realizado em três fases, sendo: Fase 1: Elaboração de uma planilha adaptada às especificidades da usina, onde foram realizados estudos avaliando quais seriam os dados de interesse para a análise, como a estrutura de custos envolvida no sistema irrigado e a metodologia para cálculo dos benefícios; Fase 2: Coleta de dados. Foi enviada a planilha aos funcionários administrativos da usina responsáveis pelo fornecimento dos dados e devolução da planilha preenchida; Fase 3: Cálculos e análises de diferentes indicadores financeiros para o sistema de irrigação, divididas em duas etapas: 1) considerando somente produtividade; 2) considerando produtividade e longevidade.

\section{Custos Totais}

O custo total anual do sistema de irrigação, obtido pela soma dos custos fixos e variáveis, foi de $R \$ 6.537 .810,70$ ao ano. A Tabela 1 mostra a composição do custo total anual e as participações relativas dos seus componentes.

Tabela 1. Custo total anual do sistema de irrigação, separado entre componentes e participações relativas

\begin{tabular}{llcccc}
\hline & Componentes & \multicolumn{3}{c}{ Custo total anual } & Participação \\
\hline \multirow{6}{*}{ Variável } & $\mathrm{R} \$$ totais & $\mathrm{R} \$$ ha $^{-1}$ & ${\mathrm{R} \$ \mathrm{t}^{-1}}$ & $\%$ \\
& Materiais de manutenção & $150.000,00$ & 23,08 & 0,96 & 2,29 \\
& Diesel & $294.000,00$ & 45,23 & 1,88 & 4,50 \\
& Lubrificantes & $8.000,00$ & 1,23 & 0,05 & 0,12 \\
& Mão de obra & $1.995 .840,00$ & 307,05 & 12,79 & 30,53 \\
& Energia elétrica & $3.347 .908,20$ & 515,06 & 21,46 & 51,21 \\
& Equipamento & $50.250,00$ & 7,73 & 0,32 & 0,77 \\
& Transporte e montagem & $54.375,00$ & 8,37 & 0,35 & 0,83 \\
\multirow{5}{*}{ Fixo } & Casa de bomba & $1.187,50$ & 0,18 & 0,01 & 0,02 \\
& Oficina de irrigação & $23.750,00$ & 3,65 & 0,15 & 0,36 \\
& Tubulação & $612.500,00$ & 94,23 & 3,93 & 9,37 \\
\hline Total & & $6.537 .810,70$ & $1.005,82$ & 41,91 & 100,00 \\
\hline
\end{tabular}

Fonte: Dados originais da pesquisa 
Notou-se que os custos operacionais (variáveis) contabilizaram o maior impacto no custo de produção, com destaque para mão de obra e energia elétrica que somam $81,74 \%$ do custo total anual. Evidencia-se o resultado potencial que uma gestão de controle pode trazer sobre o projeto, uma vez que essa pode reduzir a necessidade de energia elétrica e mão de obra e causar grande impacto na redução de custos.

\section{Benefícios Diretos}

\section{Etapa 1}

A qualidade da cana foi considerada como constante na cana irrigada e de sequeiro, ou seja, a quantidade de $\mathrm{kg}$ de ATR por tonelada de cana foi considerada a mesma, pela dificuldade na obtenção dos dados. Portanto, o benefício da produtividade foi considerado como a multiplicação da variação em produtividade pela quantidade média de ATR na cana e pelo valor do $\operatorname{ATR}\left(R \$ \mathrm{~kg}^{-1}\right)$.

Devido à importância do preço do ATR nessas análises, todo resultado gerado apresentou os três cenários de preço de ATR. A Tabela 2, a seguir, mostra cada indicador no cálculo do benefício da produtividade, considerando uma área irrigada de 6.500 ha.

Tabela 2. Benefício da irrigação pelo aumento da produtividade e seus indicadores para três cenários de preço de Açúcar Total Recuperável [ATR] em uma usina localizada no estado de Goiás

\begin{tabular}{|c|c|c|c|c|}
\hline \multirow{2}{*}{ Indicadores } & \multirow{2}{*}{ Unidade } & \multicolumn{3}{|l|}{ Cenários } \\
\hline & & Médio & Otimista & Pessimista \\
\hline Produtividade média sequeiro & t ha $^{-1}$ & 102,00 & & \\
\hline Produtividade média irrigado & $\mathrm{t} \mathrm{ha}^{-1}$ & 116,0 & & \\
\hline Delta produtividade & $\mathrm{t} \mathrm{ha}^{-1}$ & 14,00 & & \\
\hline ATR & $\mathrm{kg} \mathrm{t}^{-1}$ & 129,40 & & \\
\hline Valor do ATR & $\mathrm{R} \$ \mathrm{~kg}^{-1}$ & 0,5923 & 0,6731 & 0,51140 \\
\hline Benefício & $\mathrm{R} \$ \mathrm{ha}^{-1}$ & $1.072,97$ & $1.219,48$ & 926,46 \\
\hline Benefício & $R \$ t^{-1}$ & 76,64 & 87,11 & 66,18 \\
\hline Benefício produtividade total & $\mathrm{R} \$$ & $6.974 .288,09$ & $7.926 .588,96$ & $6.021 .987,23$ \\
\hline
\end{tabular}

Fonte: Resultados originais da pesquisa

Nessa primeira análise, em que se considerou apenas o benefício da produtividade, foi alcançada uma superioridade em relação aos custos para o cenário médio ( $R \$ 4,73 / t)$ e otimista $(R \$ 15,20 / t)$. A Tabela 1 demonstra o comparativo benefício de produtividade e custos do projeto. O resultado obteve margem de lucro de $6,18 \%$, demonstrando-se rentável. 
Tabela 1. Comparativo do benefício do aumento da produtividade com os custos da irrigação no cenário médio em uma usina no estado de Goiás

\begin{tabular}{llll}
\hline Comparação benefícios custos & $\mathrm{R} \$$ & $\mathrm{R} \$ \mathrm{ha}^{-1}$ & ${\mathrm{R} \$ \mathrm{t}^{-1}}^{-1}$ \\
\hline Benefício produtividade & $6.968 .265,19$ & $1.072,04$ & 76,64 \\
Custo & $6.537 .810,70$ & $1.005,82$ & 71,91 \\
Lucro & $430.454,49$ & 66,22 & 4,73 \\
\hline Lucro (\%) & 6,18 & & \\
\hline Fonte: & & &
\end{tabular}

Fonte: Resultados originais da pesquisa

A Figura 2 demonstra os resultados da irrigação, considerando apenas 0 benefício da produtividade.

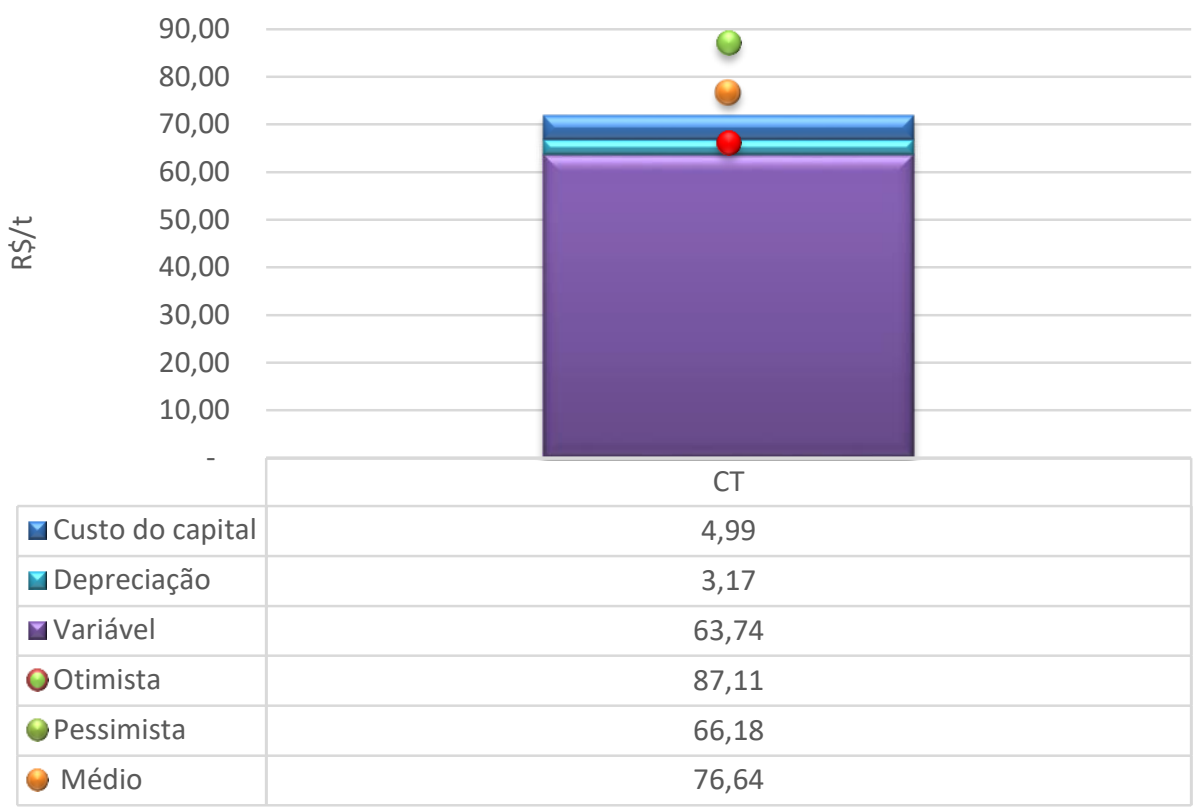

Figura 2. Resultado do benefício da produtividade em três cenários de Açúcar Total Recuperável [ATR] em uma usina no estado de Goiás

Fonte: Resultados originais da pesquisa

Os pontos na Figura 2 indicam os benefícios em seus três cenários de valor de ATR e as barras a somatória de custos variáveis (operacional), depreciações e custo do capital. Analisando os diferentes cenários de ATR, a rentabilidade da prática de irrigação foi elevada para o cenário médio e otimista, superando os custos de depreciação e custo de capital. As margens econômicas nos cenários pessimista, médio e otimista foram de $-8,66 \%, 6,18 \%$ e $17,45 \%$, respectivamente. A relação benefício/custo encontrada nessa etapa foi de 1,066 .

\section{Etapa 2}

Também pode-se apontar como benefício a longevidade do canavial. Para estimar o benefício da longevidade, considerou-se a redução do impacto da depreciação na formação do canavial. O custo de formação do canavial na região foi $R \$ 7.238,00$ ha $^{-}$ ${ }^{1}$ (PECEGE, 2015). Para o custo de formação do canavial irrigado, adicionou-se o custo 
unitário da anuidade da irrigação. A demonstração dos resultados calculados é apresentada na Tabela 4.

Tabela 4. Benefício da irrigação pelo aumento da longevidade e seus indicadores em uma usina localizada no estado de Goiás

\begin{tabular}{|c|c|c|c|}
\hline & & \multicolumn{2}{|c|}{ Benefício da longevidade } \\
\hline & & Sequeiro & Irrigado \\
\hline Longevidade & anos & 6,00 & 8,00 \\
\hline Formação do canavial & $\mathrm{R} \$ \mathrm{ha}^{-1}$ & $7.238,00$ & $7.658,98$ \\
\hline Depreciação anual & $\mathrm{R} \$ \mathrm{ha}^{-1}$ & 1206,33 & 957,37 \\
\hline Depreciação total anual & $\mathrm{R} \$$ & $7.841 .166,67$ & $6.222 .917,50$ \\
\hline Benefício total anual longevidade & $\mathrm{R} \$$ & \multicolumn{2}{|c|}{$1.618 .249,17$} \\
\hline Benefício anual longevidade & $\mathrm{R} \$ \mathrm{ha}^{-1}$ & \multicolumn{2}{|c|}{248,96} \\
\hline Benefício anual longevidade & $\mathrm{R} \$ \mathrm{t}^{-1}$ & \multicolumn{2}{|c|}{17,80} \\
\hline
\end{tabular}

Fonte: Resultados originais da pesquisa

\section{Benefício Total Anual}

O impacto desse acréscimo no benefício foi analisado na Figura 3, em que foram considerados os três cenários para o valor de ATR.

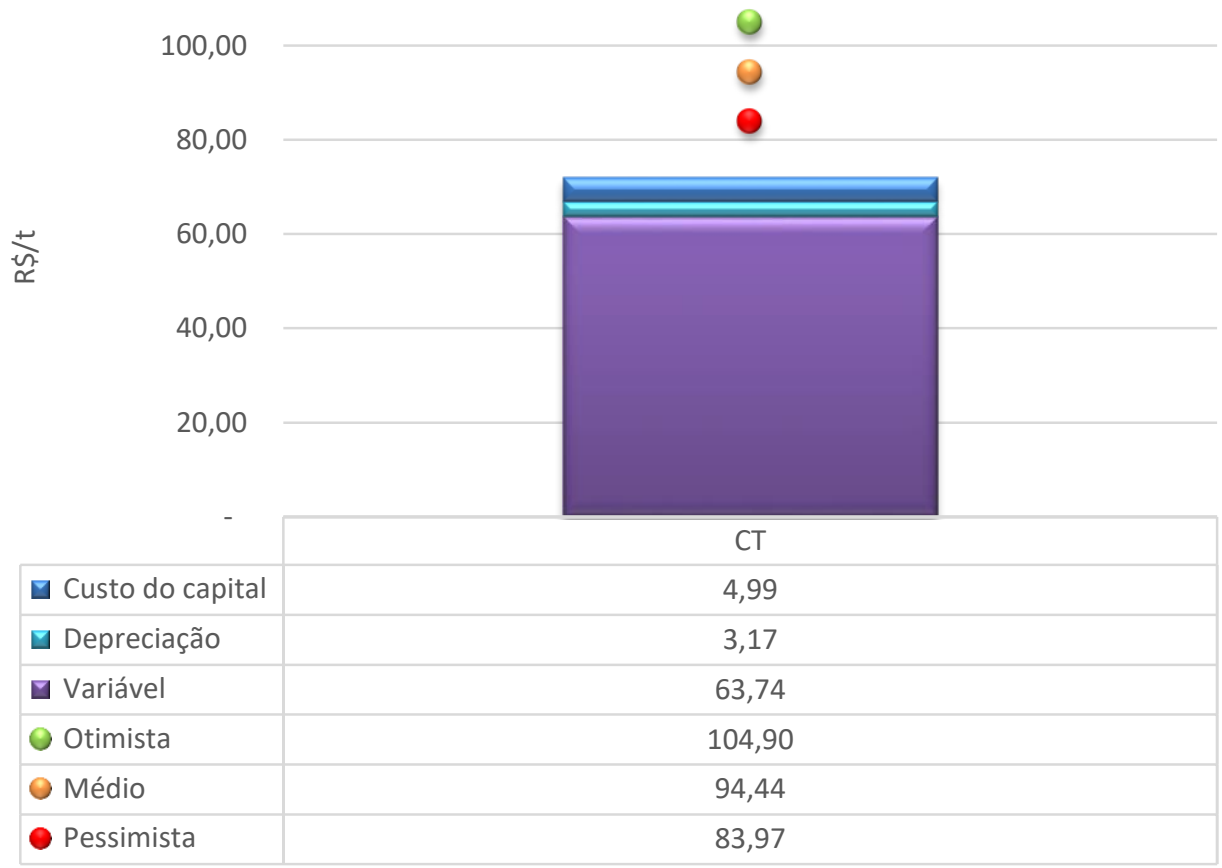

Figura 3. Resultados dos benefícios de produtividade e longevidade para três valores de Açúcar Recuperável Total [ART] em uma usina localizada no estado de Goiás Fonte: Resultados originais da pesquisa

O benefício da longevidade contabilizou como $23,2 \%$ do benefício direto anual. O impacto desse benefício alterou as margens dos cenários pessimista, médio e otimista para $14,37 \%, 23,86 \%$ e $31,46 \%$, respectivamente. A Tabela 5 demonstra o resultado do 
lucro considerado para o cenário médio na operação de irrigação. A relação benefício/custo encontrada nessa etapa foi de 1,313.

Tabela 5. Resultado financeiro da irrigação de uma usina de cana-de-açúcar localizada no estado de Goiás

\begin{tabular}{llll}
\hline & \multicolumn{1}{c}{$\mathrm{R} \$$} & $\mathrm{R} \$ \mathrm{ha}^{-1}$ & ${\mathrm{R} \$ \mathrm{t}^{-1}}^{-1}$ \\
\hline Benefício anual produtividade e longevidade & $8.586 .514,36$ & $1.321,00$ & 94,44 \\
Custo anual & $6.537 .810,70$ & $1.005,82$ & 71,91 \\
Lucro anual & $2.048 .703,66$ & 315,19 & 22,53 \\
\hline Lucro (\%) & 23,86 & & \\
\hline
\end{tabular}

Fonte: Resultados originais da pesquisa

\section{Benefícios Indiretos}

O acréscimo produtivo da irrigação reduziu a necessidade de incorporação de novas áreas e então foi considerada a redução no custo de arrendamento e redução de custo de produção em novas áreas. Dessa forma, avaliou-se como investimento alternativo à irrigação a incorporação de novas áreas e comparou os custos dessas alternativas. A Figura 4 demonstra o comparativo de custo.

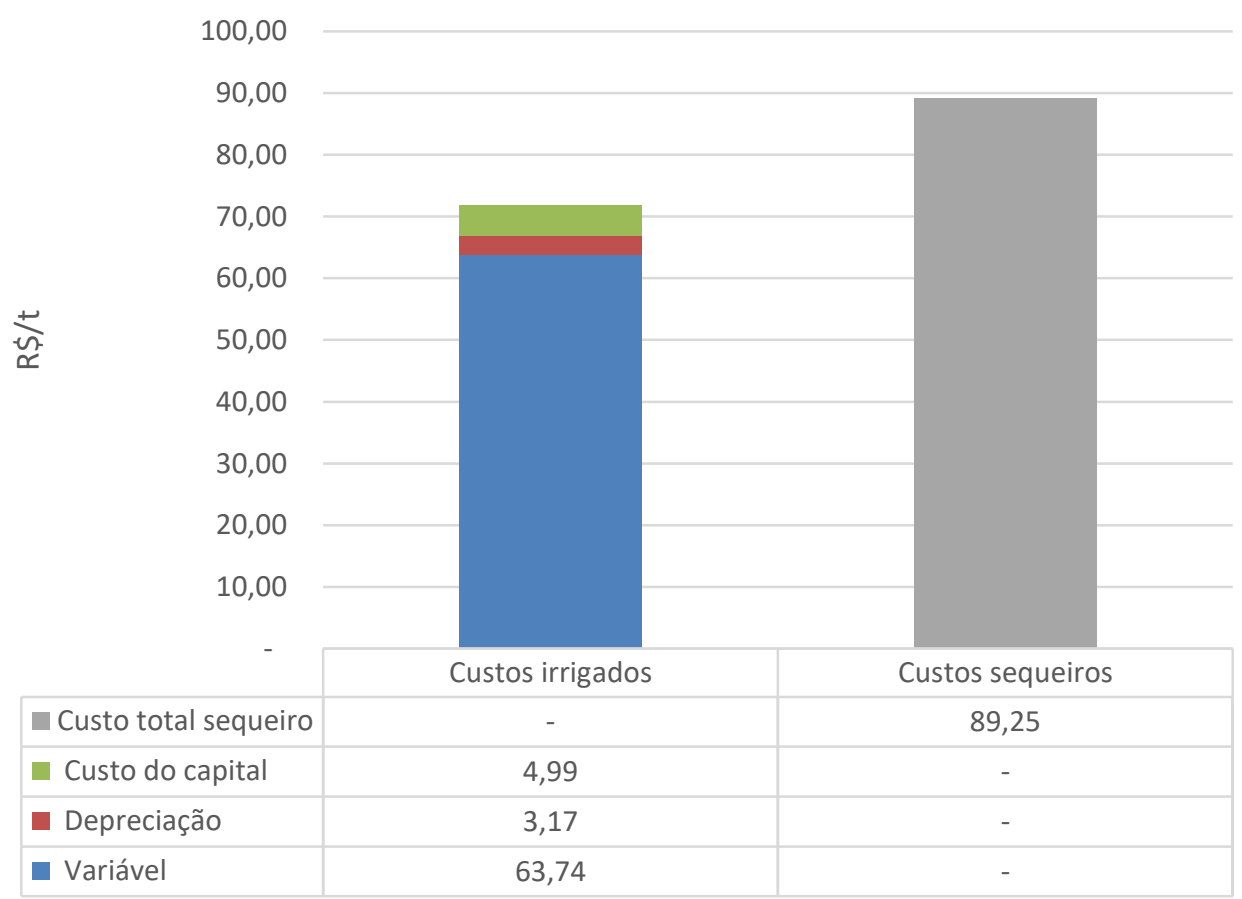

Figura 4. Comparativo de custos de produção de cana-de-açúcar entre sistemas irrigados e sequeiro em uma usina localizada no estado de Goiás Fonte: Resultados originais da pesquisa

O custo de produção de cana-própria da usina, comparado entre a irrigação de áreas existentes e a expansão para novas áreas, apresentou-se 19,4\% menor para a 
irrigação. Comparando com a aquisição de cana-de-açúcar de fornecedores, declarado $\mathrm{R} \$ 74,41$ ton $^{-1}$ em 2016, a irrigação também apresentou menor custo (Figura 4). No entanto, a cana de fornecedores apresentou teores de ATR superiores. Vale ressaltar que tanto a expansão para as novas áreas como a implantação de irrigação e a aquisição de cana-de-açúcar de fornecedores depende de diversos outros fatores e, por isso, essa é uma análise ilustrativa.

Caso esse custo total de $\mathrm{R} \$ 74,41$ ton $^{-1}$ fosse considerado como um benefício indireto adicional, somado ao benefício direto, seriam acrescentados $R \$ 9.726 .469,76$. Isso causaria aumento maior que o dobro do benefício direto da irrigação, uma vez que a relação benefício indireto/benefício direto foi de 1,13. Uma possível maneira de contabilizar esse benefício de maneira mais justa seria pela diferença entre o custo de cada sistema, nesse caso, o benefício seria de $\mathrm{R} \$ 3.188 .659,06$.

Além disso, outros benefícios indiretos podem ser considerados na irrigação de cana-de-açúcar pelas usinas, como a redução do custo de colheita para canaviais de maior produtividade, a redução no custo de transporte de cana-de-açúcar e a redução da ociosidade industrial.

\section{Conclusões}

A atividade de irrigação, demonstra elevada rentabilidade para a usina analisada. Um grande fator de vantagem da usina para essa prática é o uso da energia elétrica própria a menor custo, que permite sinergia, visto que esse componente apresenta o maior custo anual.

O custo marginal para produção da cana irrigada é de $R \$ 71,91$ por tonelada produzida, esse custo é $33 \%$ menor do que o custo da cana-própria em sequeiro que chega a $\mathrm{R} \$ 89,25$ por tonelada de cana.

Os benefícios da produtividade superam os custos para dois cenários diferentes de preço de ATR e ao se considerar o benefício da longevidade somado ao da produtividade, todos os cenários de preço de ATR são rentáveis. O resultado mostram benefícios médios de $R \$ 76,64$ por tonelada e $R \$ 94,44$ por tonelada para as etapas 1 e 2, respectivamente.

Para uma análise mais precisa, é necessário realizar visitas à propriedade para averiguar pessoalmente a coleta de dados e certificar-se de que todas as operações foram contempladas na análise. É interessante também, buscar dados da qualidade da cana entre irrigado e sequeiro e buscar os dados de produtividade e qualidade específico para as áreas com irrigação, e não os dados gerais agrícolas da usina.

Os benefícios indiretos devem ser analisados cuidadosamente, já que não há uma metodologia consolidada para sua análise, podendo variar de acordo com as 
especificidades de cada usina e de cada unidade de produção (glebas arrendadas). Devem também ser discutidos quanto à forma de avaliação, uma vez que a soma dos benefícios pode superestimar o projeto. Por exemplo, uma usina que possua a produção abaixo da capacidade de moagem, beneficiará com a irrigação na redução da ociosidade industrial pois absorverá todo o aumento de produção sem alterar sua área de produção. Já no caso de uma usina que possua produção próxima à capacidade máxima de moagem industrial, irá beneficiar-se do uso da irrigação pela redução da área de produção e possivelmente do custo de transporte se as áreas de irrigação forem próximas a unidade industrial. O impacto do custo de colheita em canaviais de maior produtividade também deve ser analisado.

\section{Referências}

Agrológica. 2002. Importância da irrigação no desenvolvimento do agronegócio. Engenharia Agrícola. Disponível em: < http://www.agr.feis.unesp.br/csei.pdf>. Acesso em: 20 out. 2016.

Companhia Nacional de Abastecimento [CONAB]. 2016. Cana-de-açúcar (Vol. 3). Disponível em: < http://www.conab.gov.br/OlalaCMS/uploads/arquivos/16_12_27_16_30_01_boletim_ca na_portugues_-3o_lev_-_16-17.pdf>. Acesso em: 20 out. 2016.

Companhia Nacional de Abastecimento [CONAB]. 2017. Cana-de-açúcar (Vol. 4). Disponível em:

http://www.conab.gov.br/OlalaCMS/uploads/arquivos/17_04_20_14_04_31_boletim_ca na_portugues_-_10_lev_-_17-18.pdf. Acesso em: 03 maio 2017.

Frizzone, J.A.; Júnior, A.S. de A.; Souza, J.L.M.; Zocoler, J.L. 2005. Planejamento de Irrigação: análise de decisão de investimento. Embrapa Informação Tecnológica; Brasília, Distrito Federal, Brasil.

Marques, P.A.A.; Frizzone, J.A. 2005. Modelo computacional para determinação do risco econômico em culturas irrigadas. Acta Scientiarum. Agronomy. 27(4): 719-727. Disponível em: <https://doi.org/10.4025/actasciagron.v27i4.1347>

Programa de Educação Continuada em Economia e Gestão de Empresas [PECEGE]. 2015. Custo de produção de cana-de-açúcar, açúcar, etanol e bioeletricidade no Brasil: fechamento da safra 2014/15 e acompanhamento da safra 2015/16. Piracicaba: Universidade de São Paulo, Escola Superior de Agricultura "Luiz de Queiroz", Programa de Educação Continuada em Economia e Gestão de Empresas/Departamento de Economia, Administração e Sociologia. 2015. 73p. Relatório apresentado a Confederação da Agricultura e Pecuária do Brasil [CNA]. 